\title{
IMPLEMENTATION OF AUGMENTED AND VIRTUAL REALITY TECHNOLOGIES FOR AN EDUCATIONAL AND RESEARCH SITUATION CENTER
}

\author{
Konstantin Kharin, Sergey Kuvshinov, Valentin Pryanichnikov \& Eleonora Anufrieva
}
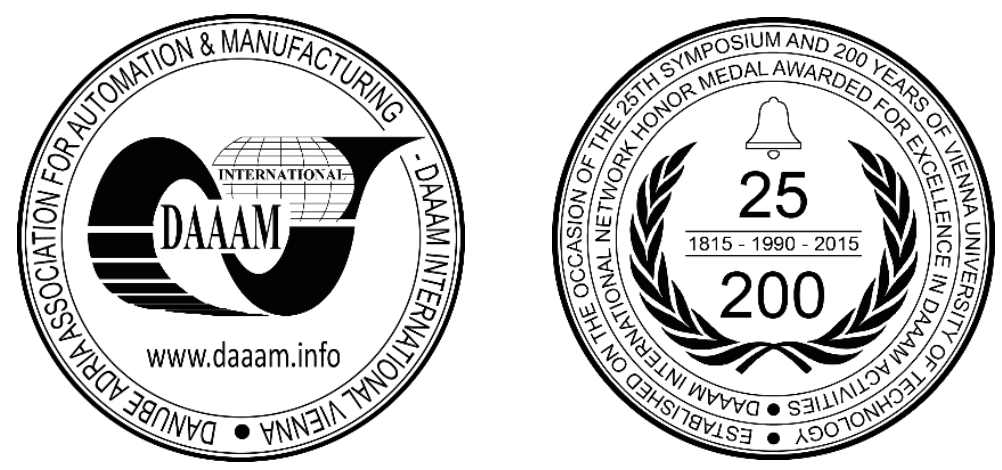

This Publication has to be referred as: Kharin, K[onstantin]; Kuvshinov, S[ergey]; Pryanichnikov, V[alentin] E[.] \& Anufrieva, E[leonora] (2017). Implementation of Augmented and Virtual Reality Technologies for an Educational and Research Situation Center, Proceedings of the 28th DAAAM International Symposium, pp.0361-0366, B. Katalinic (Ed.), Published by DAAAM International, ISBN 978-3-902734-11-2, ISSN 1726-9679, Vienna, Austria DOI: $10.2507 / 28$ th.daaam.proceedings.050

\begin{abstract}
We consider some problems in the transformation of modern education in engineering fields and the Humanities. In addition, the need for improved clarity regarding the study and analysis of complex structures and objects is concerned. Augmented and virtual reality technologies are proposed for these purposes. Some examples of application of these technologies in various educational processes are given. Experience of such application allows to conclude that they can be used to implement a variety of educational scenarios for collective and individual activities under conditions of the educational and research situation center.
\end{abstract}

Keywords: engineering education; Humanities; 3D modeling; augmented reality; virtual reality; research situation center

\section{Introduction}

This study addresses a rather narrow problem (the process of creation and development of a specific training course at an educational situation center with a multichannel presentation of 3D multimedia data and augmented and virtual reality used to synthesize and analyze the structures of complex technical objects using the example of unmanned aerial vehicles) on the one hand and the conceptual problems of technical and humanitarian education, on the other hand. Therefore, the restructuring of a specific (in our opinion, any) training course cannot be adequately understood unless the situation of the global civilizational transition is taken into account, when the former European model of training specialists has been almost exhausted, while no visible prospective model is yet available [1]. In this situation, the education system as the main mechanism of social and cultural inheritance is certainly devoid of the key pointdevelopment ideals and values; in turn, this prevents the formation of a generally valid prospective model of educational development as a whole. Most often and most convincingly, many European countries speak about insufficient funding among "their" vital problems; however, although acute, this is not the only and, at present, not the most important problem of education. 
The information, communication, and audiovisual technologies become the basis for constructing the structure of a new educational environment and organizing a new type of educational and research space: an educational and research situation center. This will allow the training of specialists in the synthesis of complex technical structures to go from the reproductive to a creative type of learning. The use of interactive information technologies as well as augmented [2] and virtual reality technologies in educational institutions provides a unique opportunity for teachers to find new ways of dealing with educational and scientific materials using the case study technology, particularly in a specially arranged audience that meets the criteria of an educational situation center [3, 4].

\section{Project culture as an effective way to creative education}

The term "project culture" is often used to characterize the culture of the entire 21 st century as its pervasive feature. For a long time, education has been developing along two noncontiguous lines (humanitarian-artistic and scientifictechnical), which currently exist as "two cultures". The conclusion about the existence of a "third culture" (project culture) is rather nontrivial; this was proposed to be called a "Design with a capital letter" and defined as "the cumulative experience of material culture and the cumulative array of experience, skills, and knowledge reflected in the art of planning, invention, creation, and implementation. Finally, it is reasonable to consider the design as a part of the general education system since the design involves fundamental cognition methods required in any activity. In other words, the design received a status of semantic activity. In the Russian professional technical literature, the concept of "design" is often identified with the implementation of a specific project, which, in turn, is focused on a final product. The practice of educational design — a kind of assembly of entire university learning — confirms, fixes, and retranslates this design ideology into the future. The transition in late 20th century to the organization of design (understood rather widely as both planning and forecasting) focused on function with the development and use of appropriate methods affects technical education quite inadequately. Discussing new approaches to engineering education and specifically the use of integrative university courses such as "Designing complex technical systems" on the example of aircraft design, one should note that all design problems can be conditionally described (simulated) by a form that governs the relationship between the research subject entities, attributes, properties, and behavior (characteristics) in an external environment. The problem has one unknown parameter (entity, environment, or characteristic), which should be determined from the remaining two components. Depending on what namely is unknown, all problems can be divided into three main classes.

The first class covers the problem of analysis when the entities are given and their characteristics (unknowns) under the conditions of a given external environment are investigated.

The second class involves synthesis problems when the required characteristics are created and one should determine the entities that provide the derivation of these characteristics under the conditions of the given environment.

The third class is called the problem of external environment assessment, when the entities and their characteristics are given and it is necessary to obtain an environment (yet unknown), such that the relevant entities manifest the given characteristics. There is no formal method that would make it possible to construct the most suitable (appropriate) model for a given problem. During the solution of the problem, the construction and subsequent correction of the model is put entirely on the man. A specific feature of the synthesis of complex object structures is the fact that the teams of people, who must interact and make decisions determining the fate of the project, create them. Therefore, it is so important now to train specialists under special conditions of an educational situation center, which develops not only skills of purely engineering activities, but also simulation and communication skills, and forms a sense of responsibility for making certain decisions.

Historically, the aviation education in many countries developed following the lead of industry together with scientific research institutes and design bureaus. The principle of professional knowledge transfer "from hand to hand" was rather efficient. In other words, the "knowledge orientation" of higher professional education as an absolute value recognized the content of appropriate scientific rather than even educational discipline, and the quality of teaching depended largely by the extent that the content of an educational subject approximates the corresponding scientific field. The structuring of the content of the subject studied and the educational process was determined by the logic of science rather than teaching. The methodological and especially psychological components of the pedagogical process were intuitive and one-sided (from teacher to student) character.

The current professional education based on both internal processes and the general course of scientific-technical and social and cultural development differs radically from the "classical" period. First of all, it became quite obvious that the activities in science and engineering and the relevant teaching are two fundamentally different types of activities. Their subjects and methods are not the same. These activities require completely different skills, dispositions, and motives. What they have in common is the information support. Here, the old division of knowledge into educational and scientific fields has lost its informational sense and acquired a new (methodical) sense. Secondly, it became obviously clear that the student couldn't be further regarded as a "black box" because the "pedagogical effect" cannot be adequately assessed. Thirdly, it is also clear that the teacher's role in the educational process changes both psychologically and conceptually. The teacher turns from the source (which often is almost unique) of knowledge into the organizer of the educational process, and his activities are directed to the learning of tools, methods, and the design 
culture in general, rather than to the design object (unlike the "designer"). It can be seen from the context that the design tools and methods likewise should not be absolutized in the educational process, which aims mainly to develop skills and abilities for independently choosing and justifying the design goals and finding the optimal ways for their achievement.

In this connection, one of the first tasks of formulating the design course and determining its structure should probably be resolved from the logic of teaching of "design rules" and the specific features of students' psychological perception, rather than from scientific and designer (mainly information-educative) logic of aircraft structure and operation. The teacher formulates (rather than creates, of course) and structures the game rules, teaches them (with individual control over their assimilation), and, if necessary, demonstrates their applicability. All this requires condition s for training specialists that are absolutely different from present-day computer classes and become increasingly more like situational centers. Fourthly, the reasoning (in a special sense of this word) begins only when consciousness is confronted with a contradiction that cannot be resolved by ready-made schemes, recipes, and algorithms. Therefore, the educational process (understood as the organization of students' reasoning) must be built as a specially organized system of possible contradictions (obstacles) in the implementation of a target (as opposed to a systematized description of ready-made (correct) solution sets). In other words, it is heuristically more important to study different errors and ways to overcome them than to study only the positive experience. Finally, the availability and remarkably rapid development of new information and computer technologies sharply (to a minimum) reduced the potential of the classical education model. Here, the question is not whether to use or not to use these technologies and not even how to use them, but how to find a new spatial and temporal model of the educational process such that any development of new technologies would only expand the capabilities of the pedagogical process.

\section{Educational and research situation center based on AR technology}

An example is the innovative Russian product that is based on the technology of augmented reality: the EligoVision interactive system [5]. The preparation of experts in the synthesis of complex technical structures requires a spatial representation of future objects and their systems, not to mention the complex processes that cannot be adequately understood in a planar representation. Under conditions of the educational situation center, the EligoVision interactive system is a set of audiovisual computer devices for data display, equipped with collective remote communication tools. This set allows the case runner, the teacher, and the entire group of students to be simultaneously involved in intensive activities, the voluminous graphical material to be handled in both 2D and 3D modes. The skills of collective communication for the synthesis of complex structures to be developed, and, which is especially important, a decision adequate to the given problem to be made.

The development of practical technologies for the educational process that emulates the situational center operation became possible in the International Institute of New Educational Technologies, which became a unique experimental platform to analyze and implement most advanced educational technologies in Russia.

The multifunctional audience Visionarium has a unique visualization system developed by the EligoVision company, and this new equipment is currently used for interactive lessons. There have been master classes for the teaching staff of both technical and humanitarian universities.

A new technique was proposed for the presentation and analysis of educational materials on the design and management of projects for creating complex technical systems, design and construction of aircrafts, etc.; conceptual teaching techniques were developed on the basis of collective and remote control of symbolic, graphical, and threedimensional applications. For example, even all existing training courses and lectures prepared in traditional presentation programs with augmented and virtual reality technologies turn into an interactive discussion and a collaboration.

\section{Implementations}

The implementation of augmented reality technologies in education began a decade ago and has been developed with an explosive behavior. The elements of augmented reality are integrated into various educational and developmental tools, including children's coloring books, textbooks on school disciplines (usually, in natural sciences), and handouts for classes on specific topics. However, most applications of this technology have a "game" and fragmentary character and therefore, are rarely used in professional and higher education.

The experience of the authors in using digital technologies in general and augmented and virtual reality in particular allows us to state that it is these educational approaches that will govern the most prospective use of augmented reality in various fields of knowledge (both engineering and humanitarian). On the one hand, this is explained by the large number of topics, phenomena, processes, etc., which description by traditional teaching methods encounters problems in visualization. 
On the other hand, the widespread use of 3D technologies, simulation, and design makes it possible to use the results of students themselves and the equipment used to increase the vividness of the given disciplines, including in the research and design activities. Often, these projects allow students to create their own 3D models and entire virtual worlds that can be used to create appropriate software. Thus, the learners themselves are engaged as "experts" and "knowledge engineers", which significantly increases their motivation in obtaining results and mastering new technologies [6].

An example of completed projects is the above-mentioned course of aircraft design and construction. The authors participated in all stages of this extensive course to develop a smart-scheme (a methodical teaching manual) that allows the student to focus on creative and personalized illustration of his own perception, existing supplementary schematics, diagrams, graphs, etc., rather than on accelerated and automatic recording of lecture fragments.

An important supplement to this manual was the augmented reality application that makes it possible to visualize most illustrations available in textbooks and smart-scheme with the help of animated 3D models that significantly increase the vividness of materials. In this case, a nonmarking technology was used that allows the illustrations themselves to be used as markers for optical tracking, including the supplements introduced during the smart-scheme completion. In the next step, the models were visualized by using modern 3D visualization systems, including MotionParallax3D technologies (virtual holograms) [7], rather than the flat screen of smartphones or tablets (see Fig. 1).

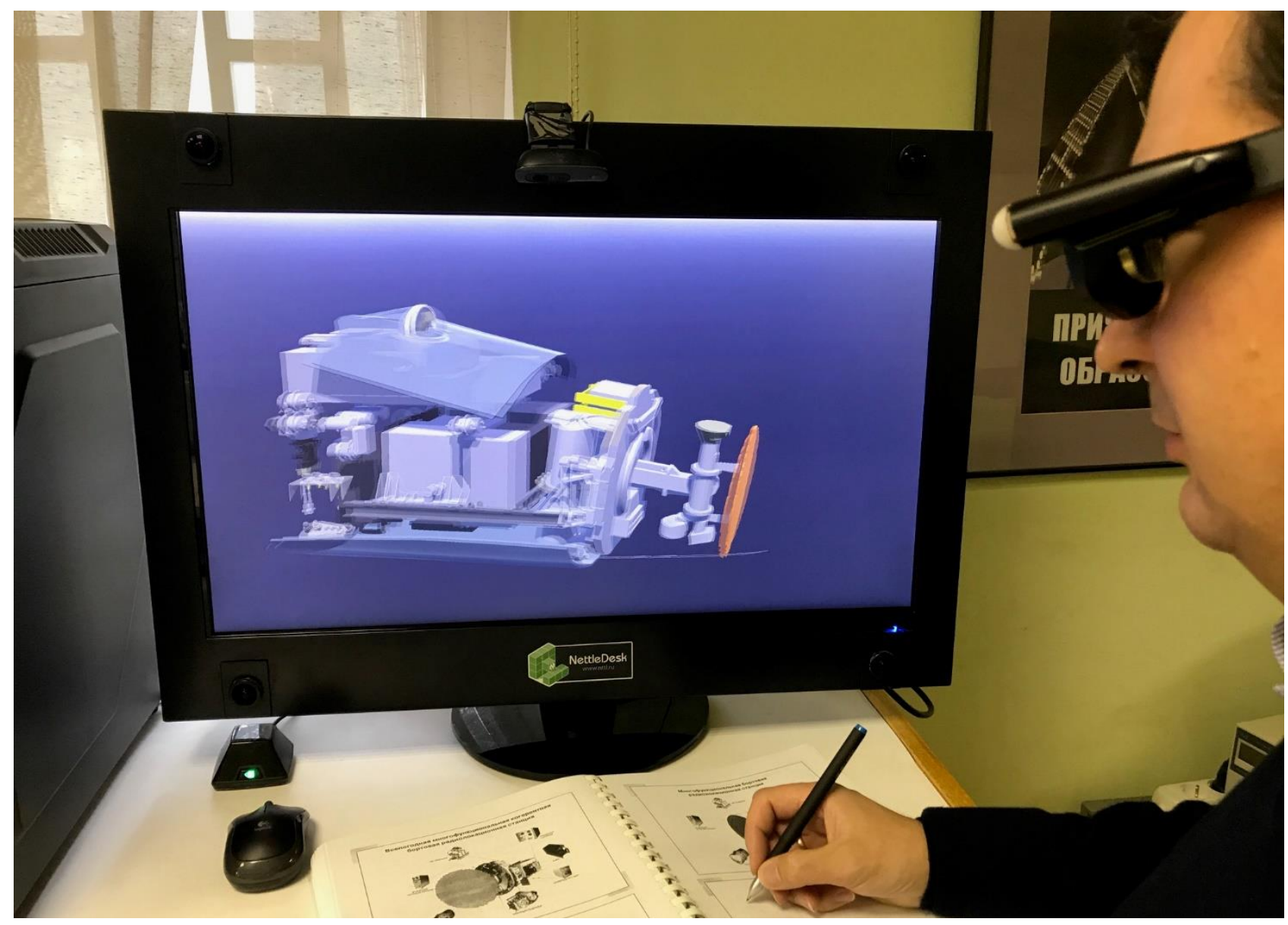

Fig. 1. Working with MotionParallax3D display

Another project was the preparation of materials for a professional course on oil production technologies. Here, the specific feature of the augmented reality technology was that the layout of drilling platforms, oil production and pumping equipment, etc. printed on a 3D printer were used as a marker for recognition in the application (see Fig. 2).

The superposition of additional information layers (geological, technological, etc.) together with the layout inspection from different angles significantly enhances the informative content of the educational material. 


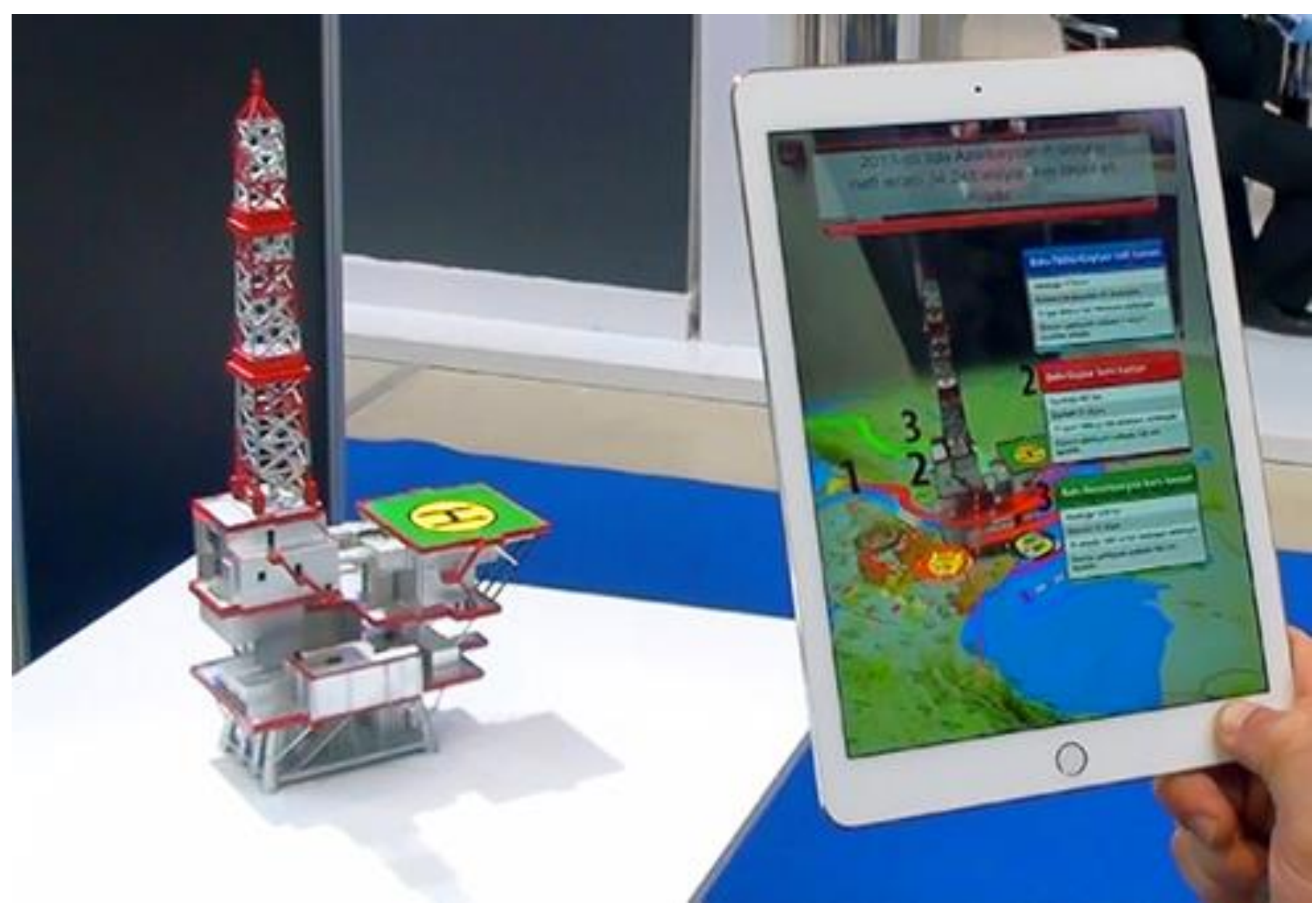

Fig. 2. AR application with a real object as a marker (drilling platform model)

The augmented reality technology can also be widely used for training in the fields of robotics, mechatronics, and sensorics. The combination of the complexity of visualization of many interacting devices and components with the study of programming, 3D modeling and design, and computer vision systems by most students [8] makes it possible to create integrated learning materials with the help of augmented reality by the students themselves during the completion of their qualification works or individual research projects. Here, in terms of the technology itself, the marking of individual components of devices, the pattern of conductor wiring or the arrangement of parts on the printed-circuit board, as well as specially designed markers (QR-codes) on the structural elements can be used as augmented reality markers.

Equally important is the use of the augmented reality technology in the humanitarian fields. An interesting example can be the learning in the conservative field of manual musical conducting. In some cases, the traditional visual method of teaching to conducting (do as I do) hinders the development of the musician creativeness. The learning process in the class of conducting is to form qualities that are professionally required for the musician and can allow him to become the leader of the performing team [9]. At the initial stage, both augmented and virtual reality technologies can be used as a developing approach to teaching to conducting. In the practice of teaching, the space of physical activities by the conductor is commonly represented as a cube since the graphics of the manual process is three-dimensional (width, height, and depth). The very concept of the conductor space is associated with a specific representation of the location of his hands in the low (the bottom of the cube), middle, and high (the upper plane of the cube) positions; in the near (front wall), middle (the cube center), and deep (rear wall) planes, and the horizontal movement of arms kind of connects the lateral planes of the cube. The gesture parameters and direction reflect the sound character, the degree of its saturation, the dynamic brightness, and specific features of the articulatory character of the movement (the "gait" of the sound stream). The initial possession of manual skills is associated with the formation of the ability to freely handle with conductor's three-dimensional space. It seems possible and prospective to use the traditional technique of mastering the conductor gesture on the basis of physical exercises seems in combination with audiovisual software and hardware tools recording diverse pieces of classical music. The use of augmented reality with markers as fragments of music score notation make it possible to read them with the help of a camera, process by a software application coupled with the reproduced music fragment, and interpret as a referenced real time video stream for displaying on a monitor or similar $2 \mathrm{D}$ or $3 \mathrm{D}$ devices. However, this is a somewhat static method that lacks to incorporate the emotional and tempo characteristics of musical works. The capabilities of the system can be expanded in the future by a rather complex system for automated analysis of professional conductor images with the translation of his gestures into a language of formal simplified description correlated to the phonogram. The comparison of the resulting generated texts will allow one to reveal the specific features of conducting or even to preliminary assess the trainee against the background of teacher's 
reference movements. The technical support for this can be realized, for example, on the basis of Kinekt-type contactless sensor controllers.

One should also note the use of augmented reality technologies in museum exposition and exhibition activities that has been actively developing in the last decade. Against the background of a large number of more or less successful examples, the authors developed and implemented the concept of multimedia expansion of the exhibition space, where the real exhibits are complemented by the demonstration of their 3D models on personal devices of visitors with the help of augmented reality and as virtual holograms in the stereo mode on devices with the MotionParallax $3 D$ technology. The modern technologies make these "hybrid" exhibitions additionally attractive for the young generation. Another factor of increased interest is (as, for example, in the exhibition project "Leonardo da Vinci in 3D" implemented by the authors) that the animated 3D models from sketches of the great Renaissance master were created by schoolchildren and students engaged in interdisciplinary projects, rather than by professional designers.

\section{Conclusion}

The augmented reality technologies allow one to look at conventional things with new eyes. The interactive augmented reality and virtual reality technologies support operation in a 3D mode: with special glasses, a team member observes voluminous objects and models and can deal with them in space with the help of special interactive manipulators. These manipulators can be used to intersect geometric objects by planes, consider sections, cut and join them, and make shifts and other operations necessary to solve the given problem. Even relatively simple applications of virtual reality make it possible to "inspect" the virtual object from different angles and "look inside" it simply by changing the position of sensors or a personal device (smartphone or tablet) in a quite natural way.

A shortcoming of this technology is the risk that the educational process can be easily turned into a game, which is especially challenging in the case of younger students. This problem can be solved by a careful elaboration of both the models used and the scenarios for their use in the educational process in order to adequately channel the "game" aspect into the right direction. In prospective applications the development of intelligent modules is planned, which will process and analyze the queries and $\log$ data of students interaction with systems of augmented reality. It could result in constructing of new pedagogical models using the intelligent expert systems.

In a number of both engineering and humanitarian disciplines, this approach is not only justified but also very efficient. These technologies and devices open a new area for methodological developments. They can be used to implement a variety of scenarios of educational tasks for collective and individual activities under conditions of a new space-the educational and research situation center.

\section{Acknowledgments}

The work was carried out with the partial financial support of the RFBR, projects No. 16-07-01264, 16-07-00935

\section{References}

[1] Katalinic, B. (2010). Engineers for Knowledge Based Society, Annals of DAAAM for 2010 \& Proceedings of the 21st International DAAAM Symposium, 20-23rd October 2010, Zadar, Croatia, ISSN 1726-9679, ISBN 978-3901509-73-5, Katalinic, B. (Ed.), pp. 0001-0002, Published by DAAAM International Vienna, Vienna

[2] https://en.wikipedia.org/wiki/Augmented_reality. (2017). Augmented reality. Accessed on: 2017-10-16

[3] Andreev, V.; Karbanov, V.; Kharin, K.; Kuvshinov, S.; Poduraev, Y. and Pryanichnikov, V. (2015). Training Situation Center Based on Three-Dimensional Virtual Studio for Distributed Mobile Robotics Laboratory, in Proceedings of the 26th International DAAAM Symposium "Intelligent Manufacturing \& Automation" 21-24th October 2015, Zadar, Croatia, EU, Ed. by B. Katalinic, 2015, Vol. 26, No. 1.

[4] Pryanichnikov, V.; Andreev, V.; Bobrov, P.; Biryukova, E.; Frolov, A.; Kharin, K.; Kirsanov, K.; Kostin, A.; Kuvshinov, S.; Marzanov, Y. and Prysev, E. (2012). Scientific-educational distributed laboratory - software and hardware means, in Annals of DAAAM for 2012 \& Proceedings of the 23th international DAAAM Symposium “Intelligent Manufacturing \& Automation" 24-27th October 2012 Zadar, Croatia, 2012, vol. 23, no. 1, pp. 11751178.

[5] http://www.eligovision.ru/en/. (2017). EligoVision company. Accessed on: 2017-10-05

[6] Andreev, V.; Pryanichnikov, V.; Poduraev, Y. \& Kuvshinov, S. (2014). Education on the basis of virtual learning robotics laboratory and group-controlled robots, in 24th DAAAM Int. Symp. on Intelligent Manufacturing and Automation, 2013, Procedia Engineering, 2014, vol. 69, pp. 35-40.

[7] Kharin, K.; Kuvshinov, S. \& Pryanichnikov, V. (2016). The Use of Three-dimensional Visualization on the Basis of the MotionParallax3D Technology in the Development of Complex Technical Devices and Training of Engineers, Proceedings of the 27th DAAAM International Symposium, pp.0338-0343, B. Katalinic (Ed.), Published by DAAAM International, ISBN 978-3-902734-08-2, ISSN 1726-9679, Vienna, Austria

[8] Pavlovsky, V.E. \& Kirkov, A.Yu. (2012) Tonal acoustic communication of robots. Preprints of KIAM, No.85, 2012.

[9] Anufrieva, E.B. \& Pichugina, L.N. (2012) The initial stage of training in choral conducting. Vuzovsky vestnik, Ekaterinburg, 2012. 